Kragujevac Journal of Mathematics

Volume 40(1) (2016), Pages 91-104.

\title{
ON $\beta$-ABSOLUTE CONVERGENCE OF VILENKIN-FOURIER SERIES WITH SMALL GAPS
}

\author{
BHIKHA LILA GHODADRA ${ }^{1}$
}

\begin{abstract}
The study of absolute convergence of Fourier series is one of the most important problems of Fourier Analysis and the problem has been studied intensively by many researchers in the setting of circle group in particular and classical groups in general. Recently, in [Math. Inequal. Appl. 17 (2) (2014), 749-760], we have studied the $\beta$-absolute convergence $(0<\beta \leq 2)$ of Vilenkin-Fourier series for the functions of various classes of functions of generalized bounded fluctuation and given sufficient conditions in terms of modulus of continuity. In this paper, we prove that this is a matter only of local fluctuation for functions with the Vilenkin-Fourier series lacunary with small gaps. Our results, as in the case of trigonometric Fourier series, illustrate the interconnection between 'localness' of the hypothesis and type of lacunarity and allow us to interpolate the results.
\end{abstract}

\section{INTRODUCTION}

Let $G$ be a Vilenkin group, that is, a compact metrizable zero-dimensional (infinite) abelian group. Then the dual group $X$ of $G$ is a discrete, countable, torsion, abelian group (see [7, Theorems 24.15 and 24.26]). In 1947, N. J. Vilenkin [21] developed part of the Fourier theory on $G$ and proved an analogue of Bernstein's theorem [1, Vol. II, p. 154] concerning the absolute convergence of Vilenkin-Fourier series for a primary group $G$ [21, Theorem 5]. Later Onneweer and Waterman [8-11] introduced various classes of functions of bounded fluctuations and studied the convergence problems for functions of these classes. Interestingly, Onneweer [8, Corollary 2] proved an

Key words and phrases. Hölder's inequality, Jensen's inequality, Wiener-Ingham inequality, function of generalized bounded fluctuation, Vilenkin-Fourier series, $\beta$-absolute convergence, lacunary series with small gaps.

2010 Mathematics Subject Classification. Primary: 42C10. Secondary: 26D15, 43A25, 43A40, $43 \mathrm{~A} 75$.

Received: November 9, 2015.

Accepted: February 12, 2016. 
analogue of Bernstein's theorem for any bounded Vilenkin group and an analogue of Zygmund's theorem [1, Vol. II, p. 161] for functions of $p$-generalized bounded fluctuation $(1 \leq p<2)$ defined on any bounded Vilenkin group [8, Corollary 3]. Onneweer continued study further and in his second paper he obtained a sufficiency condition in terms of $n$-th integral modulus of continuity of order $p$ of a function $f \in L^{p}(G)$ to be in $\mathrm{A}(\beta)[9$, Theorem 1] and derived an analogue of Szász's theorem [19] from it. Vilenkin and Rubinstěin [22] proved an analogue of a well-known theorem Stečhkin [18]. Quek and Yap [14] then extended above results of Onneweer to arbitrary Vilenkin groups and Uno [20] proved an analogue of a circle group result of Schramm and Waterman [16, Theorem 1] for any Vilenkin group. In [4], for any bounded Vilenkin group, we have generalized the result of Uno [4, Theorem 1] and also proved a similar result for functions in the class of $\phi$ - $\Lambda$-generalized bounded fluctuation [4, Theorem 2]. Further, in [6], we have proved an analogue of the Wiener-Ingham inequality (see [6, Theorem 2]) and as its applications, extended the analogues on a Vilenkin group $G$ of the well-known results of Bernstěin, Zygmund, Szász, and Stečhkin concerning the absolute convergence of Fourier series on $G$ obtained by Vilenkin and Rubinstěin [22], Onneweer [9], and Quek and Yap ([14, 15]) for the lacunary Fourier series on $G$. In this paper, we prove that this is a matter only of local fluctuation for functions with the Vilenkin-Fourier series lacunary with small gaps. As in the case of trigonometric Fourier series (see [12]) and of our earlier results for Vilenkin-Fourier series (see $[5,6]$ ), here also we give an interconnection between the 'type of lacunarity' in Vilenkin-Fourier series and the localness of the hypothesis to be satisfied by the generic functions, which allow us to interpolate results concerning $\beta$-absolute convergence of lacunary and non-lacunary Vilenkin-Fourier series.

\section{Notation And Definitions}

For $G$ and $X$ as above, Vilenkin [21, Sections 1.1, 1.2] proved the existence of a sequence $\left\{X_{n}\right\}$ of finite subgroups of $X$ and of a sequence $\left\{\varphi_{n}\right\}$ in $X$ such that the following hold:

(i) $X_{0}=\left\{\chi_{0}\right\}$, where $\chi_{0}$ is the identity character on $G$;

(ii) $X_{0} \subset X_{1} \subset X_{2} \subset \cdots$;

(iii) for each $n \geq 1$, the quotient group $X_{n} / X_{n-1}$ is of prime order $p_{n}$;

(iv) $X=\bigcup_{n=0}^{\infty} X_{n}$;

(v) $\varphi_{n} \in X_{n+1} \backslash X_{n}$ for all $n \geq 0$;

(vi) $\varphi_{n}^{p_{n+1}} \in X_{n}$ for all $n \geq 0$.

The group $G$ is bounded if

$$
p_{0}=\sup _{i=1,2, \ldots} p_{i}<\infty
$$

otherwise, $G$ is said to be unbounded. Using the $\varphi_{n}$ 's, we can enumerate $X$ as follows. Let $m_{0}=1$, and let $m_{n}=\prod_{i=1}^{n} p_{i}$ for $n \in \mathbb{N}$. Then each $k \in \mathbb{N}$ can be uniquely 
represented as $k=\sum_{i=0}^{s} a_{i} m_{i}$ with $0 \leq a_{i}<p_{i+1}$ for $0 \leq i \leq s$; we define $\chi_{k}$ by the formula $\chi_{k}=\varphi_{0}^{a_{0}} \cdots \varphi_{s}^{a_{s}}$.

$G=\prod_{n=1}^{\infty} \mathbb{Z}_{p_{n}},\left\{p_{n}\right\}$ being a sequence of prime numbers, is a standard example. If $p_{n}=2$ for all $n, X$ is the group of Walsh functions $\psi_{n}, n=0,1,2, \ldots$, and $X_{n}=\left\{\psi_{0}, \psi_{1}, \ldots, \psi_{2^{n}-1}\right\}$ (using Payley enumeration; see [13]) described by N. J. Fine [3]. If $p_{n}=p$ for all $n, X$ is the group of generalized Walsh functions [2].

Let $d x$ or $m$ denote the normalized Haar measure on $G$. For $f \in L^{1}(G)$, the Vilenkin-Fourier series of $f$ is given by

$$
S[f](x)=\sum_{n=0}^{\infty} \hat{f}(n) \chi_{n}(x), \quad \hat{f}(n)=\int_{G} f(x) \bar{\chi}_{n}(x) d x,
$$

where $\hat{f}(n)(n=0,1,2, \ldots)$ is the $n$th Vilenkin-Fourier coefficient of $f$. It is said to be $\beta$-absolutely convergent, where $\beta$ is a positive real number, if $\sum_{n=0}^{\infty}|\hat{f}(n)|^{\beta}<\infty$. In this case we write $f \in \mathrm{A}(\beta)$ and we shall denote $\mathrm{A}(1)$ by A. Further, the VilenkinFourier series (2.1) is said to be lacunary with small gaps if $\hat{f}(n)=0$ for $n \neq n_{k}$, where $\left\{n_{k}\right\}_{k=1}^{\infty}$ is an increasing sequence of positive integers satisfying the small gap condition

$$
\left(n_{k+1}-n_{k}\right) \geq q \geq 1, \quad k=1,2, \ldots,
$$

or, in particular, a more stringent small gap condition

$$
\left(n_{k+1}-n_{k}\right) \rightarrow \infty \text { as } k \rightarrow \infty \text {. }
$$

Observe that for each $n, X_{n}=\left\{\chi_{k}: 0 \leq k<m_{n}\right\}$. Let $G_{n}$ be the annihilator of $X_{n}$, that is,

$$
G_{n}=\left\{x \in G: \chi(x)=1, \chi \in X_{n}\right\}=\left\{x \in G: \chi_{k}(x)=1,0 \leq k<m_{n}\right\} .
$$

Then obviously, $G=G_{0} \supset G_{1} \supset G_{2} \supset \cdots, \bigcap_{n=0}^{\infty} G_{n}=\{0\}$, and the $G_{n}$ 's form a fundamental system of neighborhoods of zero in $G$ which are compact, open and closed subgroups of $G$. Further, the index of $G_{n}$ in $G$ is $m_{n}$ and since the Haar measure is translation invariant with $m(G)=1$, one has $m\left(G_{n}\right)=1 / m_{n}$. In [21, Section 3.2] Vilenkin proved that for each $n \geq 0$ there exists $x_{n} \in G_{n} \backslash G_{n+1}$ such that $\chi_{m_{n}}\left(x_{n}\right)=\exp \left(2 \pi i / p_{n+1}\right)$ and observed that each $x \in G$ has a unique representation $x=\sum_{i=0}^{\infty} b_{i} x_{i}$, with $0 \leq b_{i}<p_{i+1}$ for all $i \geq 0$. This representation of the elements of $G$ enables one to order them by means of the lexicographic ordering of the corresponding sequence $\left\{b_{n}\right\}$ and one observes that for each $n=1,2, \ldots$,

$$
G_{n}=\left\{x \in G: x=\sum_{i=0}^{\infty} b_{i} x_{i}, b_{0}=\cdots=b_{n-1}=0\right\} .
$$

Consequently, each coset of $G_{n}$ in $G$ has a representation of the form $z+G_{n}$, where $z=\sum_{i=0}^{n-1} b_{i} x_{i}$ for some choice of the $b_{i}$ with $0 \leq b_{i}<p_{i+1}$. These $z$, ordered lexicographically, are denoted by $\left\{z_{\alpha}^{(n)}\right\}\left(0 \leq \alpha<m_{n}\right)$. 
Observe also that for $l, N_{0} \in \mathbb{N}$ if $l>N_{0}$ then $G_{l} \subset G_{N_{0}}$ and therefore

$$
\begin{aligned}
G_{l} & =\left\{x \in G: x=\sum_{i=l}^{\infty} b_{i} x_{i}\right\} \\
& =\left\{x \in G_{N_{0}}: x=\sum_{i=N_{0}}^{\infty} b_{i} x_{i}, b_{N_{0}}=\cdots=b_{l-1}=0\right\} .
\end{aligned}
$$

Thus each coset of $G_{l}$ in $G_{N_{0}}$ has a representation of the form $z+G_{l}$, where $z=$ $\sum_{i=N}^{l-1} b_{i} x_{i}$ for some choice of the $b_{i}$ with $0 \leq b_{i}<p_{i+1}$. These $\left(m_{l} / m_{N_{0}}\right)=p_{N_{0}+1} p_{N_{0}+2} \cdots p_{l}=L$ (say) cosets of $G_{l}$ in $G_{N_{0}}$ are precisely the cosets $z_{\alpha}^{(l)}+G_{l}, \alpha=0,1, \ldots, L-1$, of $G_{l}$ in $G$ in that order.

Finally, we observe that for a given $y_{0}=\sum_{i=0}^{\infty} c_{i} x_{i}$ in $G$ and $N_{0} \in \mathbb{N}$, the coset $y_{0}+G_{N_{0}}$ given by

$$
y_{0}+G_{N_{0}}=\left\{x=\sum_{i=0}^{\infty} b_{i} x_{i} \in G: b_{i}=c_{i}, i=0,1, \ldots, N_{0}-1\right\}
$$

contains $y_{0}$ and is of Haar measure $1 / m_{N_{0}}$. Further, as $G_{N_{0}}$ is the disjoint union of the cosets $z_{\alpha}^{(l)}+G_{l}, \alpha=0,1, \ldots, L-1$, for $l>N_{0}$, the coset $y_{0}+G_{N_{0}}$ is the disjoint union of the cosets $y_{0}+z_{\alpha}^{(l)}+G_{l}, \alpha=0,1, \ldots, L-1$.

It may be noted that the choice of $\varphi_{n} \in X_{n+1} \backslash X_{n}$ and of the $x_{n} \in G_{n} \backslash G_{n+1}$ is not uniquely determined by the groups $X$ and $G$. In the following, it is assumed that a particular choice has been made.

Let $f$ be a complex-valued function defined on $G$, let $p \geq 1$ be a real number, let $\Lambda=\left\{\lambda_{n}\right\}$ be a non-decreasing sequence of positive real numbers such that $\sum_{n=1}^{\infty}\left(1 / \lambda_{n}\right)$ diverges, and let $\phi:[0, \infty) \rightarrow[0, \infty)$ be a strictly increasing function. Customarily $\phi$ is considered to be a convex function such that

$$
\phi(0)=0, \quad \frac{\phi(x)}{x} \rightarrow 0 \quad\left(x \rightarrow 0_{+}\right), \quad \frac{\phi(x)}{x} \rightarrow \infty \quad(x \rightarrow \infty) .
$$

Such a function is called an $N$-function. It is necessarily continuous and strictly increasing on $[0, \infty)$. For $H \subset G$, the oscillation of $f$ on $H$ is defined as

$$
\operatorname{osc}(f ; H)=\sup \left\{\left|f\left(x_{1}\right)-f\left(x_{2}\right)\right|: x_{1}, x_{2} \in H\right\} \text {. }
$$

We need the following definitions.

Definition 2.1. For a function $f: G \rightarrow \mathbb{C}$ and $n \in \mathbb{N} \cup\{0\}$, we define the $n$-th modulus of continuity of $f$ over the coset $I=y_{0}+G_{N_{0}}$ by

$$
\omega_{n}(f, I)=\sup \left\{\left|\left(T_{h} f-f\right)(x)\right|: x \in I, h \in G_{n}\right\},
$$

where $\left(T_{h} f\right)(x)=f(x+h)$, for all $x \in G$.

Definition 2.2. For a function $f: G \rightarrow \mathbb{C}, n \in \mathbb{N} \cup\{0\}$, and $1 \leq p<\infty$, we define the $n$-th integral modulus of continuity of order $p$ of $f$ over the coset $I=y_{0}+G_{N_{0}}$ by

$$
\omega^{(p)}(f, n, I)=\sup \left\{\left\|T_{h} f-f\right\|_{p, I}: h \in G_{n}\right\},
$$


where $\|(\cdot)\|_{p, I}=\left\|(\cdot) \chi_{I}\right\|_{p}$ in which $\chi_{I}$ is the characteristic function of $I$ and $\|(\cdot)\|_{p}$ denotes the $L^{p}$ norm on $G$.

When $p=\infty$ we put, $\omega^{(\infty)}(f, n, I)=\omega_{n}(f, I)$, where $\omega_{n}(f, I)$ is as in Definition 2.1. Also, when $I=G$, we omit writing $I$ and in that case $\omega_{n}(f)$ is the $n$-th modulus of continuity on $G$ as in [8, Definition 2]. Furthermore, when $I=G$, $\omega^{(p)}(f, n, I)=\omega^{(p)}(f, n)$ as in [9, Definition 1].

Definition 2.3. We say a measurable function $f$ is of:

(a) $p$ - $\Lambda$-bounded fluctuation over $y_{0}+G_{N_{0}}\left(f \in \Lambda \mathrm{BF}^{(p)}\left(y_{0}+G_{N_{0}}\right)\right)$ if the total $p$ - $\Lambda$-fluctuation of $f$ on $y_{0}+G_{N_{0}}$, given by

$$
F_{p \Lambda}\left(f ; y_{0}+G_{N_{0}}\right)=\sup \left\{\left(\sum_{n} \frac{\left(\operatorname{osc}\left(f ; I_{n}\right)\right)^{p}}{\lambda_{n}}\right)^{1 / p}\right\}
$$

is finite, where the supremum is taken over all finite disjoint collections $\left\{I_{1}, I_{2}, \ldots, I_{T}\right\}$ in which each $I_{t}$ is a coset of some $G_{m(t)}$ and $\cup_{t=1}^{T} I_{t}=y_{0}+G_{N_{0}}$;

(b) $\phi$ - $\Lambda$-bounded fluctuation over $y_{0}+G_{N_{0}}\left(f \in \phi \Lambda \mathrm{BF}\left(y_{0}+G_{N_{0}}\right)\right)$ if the total $\phi$ - $\Lambda$-fluctuation of $f$ on $y_{0}+G_{N_{0}}$, given by

$$
F_{\phi \Lambda}\left(f ; y_{0}+G_{N_{0}}\right)=\sup \left\{\sum_{n} \frac{\phi\left(\operatorname{osc}\left(f ; I_{n}\right)\right)}{\lambda_{n}}\right\}
$$

is finite, where the supremum is taken over all finite disjoint collections $\left\{I_{1}, I_{2}, \ldots, I_{T}\right\}$ in which each $I_{t}$ is a coset of some $G_{m(t)}$ and $\cup_{t=1}^{T} I_{t}=y_{0}+G_{N_{0}}$.

Definition 2.4. We say a measurable function $f$ is of:

(a) $p$ - $\Lambda$-generalized bounded fluctuation over $y_{0}+G_{N_{0}}\left(f \in \Lambda \mathrm{GBF}^{(p)}\left(y_{0}+G_{N_{0}}\right)\right)$ if the total generalized $p$ - $\Lambda$-fluctuation of $f$ on $y_{0}+G_{N_{0}}$, given by

$\Lambda G F_{p}\left(f ; y_{0}+G_{N_{0}}\right)=\sup _{l \geq N_{0}} \sup _{\alpha}\left(\sum_{j=0}^{m_{l} / m_{N_{0}}-1} \frac{\left(\operatorname{osc}\left(f ; y_{0}+z_{\alpha}^{(l)}+G_{l}\right)\right)^{p}}{\lambda_{j+1}}\right)^{1 / p}$

is finite, where $\sup _{\alpha}$ denotes the supremum over all permutations of $\left\{0,1, \ldots, m_{l} / m_{N_{0}}-1\right\}$

(b) $\phi$ - $\Lambda$-generalized bounded fluctuation over $y_{0}+G_{N_{0}}\left(f \in \phi \Lambda \mathrm{GBF}\left(y_{0}+G_{N_{0}}\right)\right)$ if the total generalized $\phi$ - $\Lambda$-fluctuation of $f$ on $y_{0}+G_{N_{0}}$, given by

$$
\Lambda G F_{\phi}\left(f ; y_{0}+G_{N_{0}}\right)=\sup _{l \geq N_{0}} \sup _{\alpha} \sum_{j=0}^{m_{l} / m_{N_{0}}-1} \frac{\phi\left(\operatorname{osc}\left(f ; y_{0}+z_{\alpha}^{(l)}+G_{N}\right)\right)}{\lambda_{j+1}}
$$

is finite, where $\sup _{\alpha}$ is as in (a) above.

We observe that when $p=1$ and $y_{0}+G_{N_{0}}=G$, the class $\Lambda \mathrm{BF}^{(p)}\left(y_{0}+G_{N_{0}}\right)$ is same as the class $\Lambda \mathrm{BF}$ of functions of $\Lambda$-bounded fluctuation on $G$ (see 
[10, Definition 2]). Also, when $\left.y_{0}+G_{N_{0}}=G, \phi \Lambda \mathrm{BF}\left(y_{0}+G_{N_{0}}\right)\right)$ is the class of functions of $\phi$ - $\Lambda$-bounded fluctuation over $G$ [10, Definition 3]. Further, if $\phi(x)=x^{p}(p \geq 1)$, then $\phi \Lambda \mathrm{BF}\left(y_{0}+G_{N_{0}}\right)=\Lambda \mathrm{BF}^{(p)}\left(y_{0}+G_{N_{0}}\right)$ and $\phi \Lambda \mathrm{GBF}\left(y_{0}+G_{N_{0}}\right)=\Lambda \mathrm{GBF}^{(p)}\left(y_{0}+G_{N_{0}}\right)$; we shall omit writing the superscript $(p)$ when $p=1$. Further, from Definition 2.3 and Definition 2.4, it is clear that $\Lambda \mathrm{BF}^{(p)}\left(y_{0}+G_{N_{0}}\right) \subset \Lambda \mathrm{GBF}^{(p)}\left(y_{0}+G_{N_{0}}\right)$ and $\phi \Lambda \mathrm{BF}\left(y_{0}+G_{N_{0}}\right) \subset \phi \Lambda \mathrm{GBF}\left(y_{0}+G_{N_{0}}\right)$.

\section{Statements of Results}

We prove the following results.

Theorem 3.1. Let $G$ be bounded, $f \in L^{1}(G)$ possess a lacunary Vilenkin-Fourier series

$$
\sum_{k=1}^{\infty} \hat{f}\left(n_{k}\right) \chi_{n_{k}}(x)
$$

with small gaps (2.2), and let $I=y_{0}+G_{N_{0}}$ be the coset with Haar measure $1 / m_{N_{0}} \geq$ $1 / q$. If $f \in \Lambda \mathrm{GBF}^{(p)}(I), 1 \leq p<2 r, 1 \leq r<\infty, 0<\beta \leq 2$, and

$$
\sum_{n=N_{0}}^{\infty}\left\{\left[\frac{\left(\omega^{(p+(2-p) s)}(f, n, I)\right)^{2-p / r}}{\left(\sum_{j=1}^{m_{n} / m_{N_{0}}} \frac{1}{\lambda_{j}}\right)^{1 / r}}\right]^{\beta / 2} \sum_{\substack{k \\ m_{n} \leq n_{k}<m_{n+1}}} \frac{1}{k^{\beta / 2}}\right\}<\infty,
$$

in which $1 / r+1 / s=1$, then

$$
\sum_{k=1}^{\infty}\left|\hat{f}\left(n_{k}\right)\right|^{\beta}<\infty
$$

Remark 3.1. Since $\Lambda \mathrm{BF}^{(p)}(I) \subset \Lambda \mathrm{GBF}^{(p)}(I)$, Theorem 3.1 obviously holds for functions in $\Lambda \mathrm{BF}^{(p)}(I)$ also.

When the Fourier series is non-lacunary, taking $n_{k}=k$ for all $k$ and $I=G$ in Theorem 3.1 we obtain

Corollary 3.1. Let $1 \leq r<\infty$ and $1 \leq p<2 r$. If $f \in \Lambda \mathrm{GBF}^{(p)}(G)$ satisfies

$$
\sum_{n=0}^{\infty}\left[\frac{\left(m_{n}\right)^{2 / \beta-1}\left(\omega^{(p+(2-p) s)}(f, n)\right)^{2-p / r}}{\left(\sum_{j=1}^{m_{n}} \frac{1}{\lambda_{j}}\right)^{1 / r}}\right]^{\beta / 2}<\infty
$$

in which $1 / r+1 / s=1$, then $f \in A(\beta)$ for $0<\beta \leq 2$. 
Theorem 3.2. Let $G, f$, and $I$ be as in Theorem 3.1. If $f \in \phi \Lambda \mathrm{GBF}(I), 1 \leq p<2 r$, $1 \leq r<\infty, 1 / r+1 / s=1$, and

$$
\sum_{n=N_{0}}^{\infty}\left\{\left[\left\{\phi^{-1}\left(\frac{\left(\omega^{(p+(2-p) s)}(f, n, I)\right)^{2 r-p}}{\sum_{j=1}^{m_{n} / m_{N_{0}}} \frac{1}{\lambda_{j}}}\right)\right\}^{1 / r}\right]^{\beta / 2} \sum_{\substack{k \\ m_{n} \leq n_{k}<m_{n+1}}} \frac{1}{k^{\beta / 2}}\right\}<\infty
$$

then (3.2) holds, in which $\phi$ is a $\Delta_{2}$-function (that is, there is a constant $d \geq 2$ such that $\phi(2 x) \leq d \phi(x)$, for all $x \geq 0)$.

Remark 3.2. Since $\phi \Lambda \mathrm{BF}(I) \subset \phi \Lambda \mathrm{GBF}(I)$, Theorem 3.2 obviously holds for functions in $\phi \Lambda \mathrm{BF}(I)$ also.

Corollary 3.2. If $f \in \phi \Lambda \operatorname{GBF}(G), 1 \leq p<2 r, 1 \leq r<\infty, 1 / r+1 / s=1$, and

$$
\sum_{n=0}^{\infty}\left[\left(m_{n}\right)^{2 / \beta-1}\left\{\phi^{-1}\left(\frac{\left(\omega^{(p+(2-p) s)}(f, n)\right)^{2 r-p}}{\sum_{j=1}^{m_{n}} \frac{1}{\lambda_{j}}}\right)\right\}^{1 / r}\right]^{\beta / 2}<\infty
$$

then $f \in A(\beta)$ for $0<\beta \leq 2$, in which $\phi$ is a $\Delta_{2}$-function.

Remark 3.3. Theorems 3.1 and 3.2 are Vilenkin group analogues of the corresponding circle group results of Vyas [23, Theorem 1.1] and [24, Theorem 1.1] respectively.

Remark 3.4. Observe that $n_{k}=k$ for all $k \Longrightarrow q=1$ in $(2.2) \Longrightarrow I$ is of Haar measure 1 in above theorems $\Longrightarrow I=G$; and one gets above Corollaries 3.1 and 3.2 which are corresponding results for non-lacunary Vilenkin-Fourier series (see [4, Theorems 1 and $2]$ ). On the other hand, if the Vilenkin-Fourier series (3.1) of $f \in L^{1}(G)$ has gaps (2.3) then above results hold if the coset $I$ is just of positive measure. Because if $|I|>0$, by the form of $I,|I|=1 / m_{N_{0}}$ where $N_{0} \in \mathbb{N}$ can be taken as large as required. In view of (2.3), one gets $\left(n_{k+1}-n_{k}\right) \geq m_{N_{0}}$ for all $k \geq k_{0}$ for a suitable $k_{0}=k_{0}\left(N_{0}\right)$. Then adding to $f(x)$ the Vilenkin polynomial $\sum_{j=1}^{k_{0}}\left(-\hat{f}\left(n_{j}\right)\right) \chi_{n_{j}}(x)$ one gets a function $g$ whose Vilenkin-Fourier series is lacunary of the form (3.1) having gaps (2.2) with $q=m_{N_{0}}$ and results are true for $g$. Since $f$ and $g$ differ by a polynomial, results are true for $f$ as well. Our results thus interpolates lacunary and non-lacunary results concerning $\beta$ absolute convergence of Vilenkin-Fourier series - displaying beautiful interconnection between types of lacunarity (as determined by $q$ in (2.2)) and localness of hypothesis to be satisfied by the generic function (as determined by the $q$-dependent length of $I$ ).

\section{Proof of the Results}

The following lemmas are needed.

Lemma 4.1 ([22, p. 5]). For each $N=0,1,2, \ldots$, and $k \geq m_{N}$ we have

(a) $\int_{G_{N}} \chi_{k}(h) d h=0$;

(b) $\int_{G_{N}}\left|\chi_{k}(h)-1\right|^{2} d h=2 \int_{G_{N}}\left[1-\operatorname{Re} \chi_{k}(h)\right] d h=2\left|G_{N}\right|=2 / m_{N}$. 
Lemma 4.2 ([17, Lemma 2]). If $u_{n} \geq 0$, for $n \in \mathbb{N}, u_{n} \not \equiv 0$ and a function $F(u)$ is concave, increasing, and $F(0)=0$, then

$$
\sum_{n=1}^{\infty} F\left(u_{n}\right) \leq 2 \sum_{n=1}^{\infty} F\left(\frac{1}{n} \sum_{k=n}^{\infty} u_{k}\right)
$$

The following lemma was proved in [6, Theorem 3.1] which gives an analogue of the Wiener-Ingham inequality [25, Vol. I, p. 222] to Vilenkin groups.

Lemma 4.3. Let $f$ and $I$ be as in Theorem 3.1. If $f \in L^{r}(I), 1<r \leq 2$, and $1 / s=1-1 / r$ then $\left|\hat{f}\left(n_{k}\right)\right| \leq|I|^{-1}|| f \|_{1, I}$ and

$$
\left(\sum_{k=1}^{\infty}\left|\hat{f}\left(n_{k}\right)\right|^{s}\right)^{1 / s} \leq|I|^{-1}|| f \|_{r, I}
$$

Proof of Main Theorem 3.1. We may assume without loss of generality that $y_{0}=0$ so that $I=G_{N_{0}}$; for, otherwise one works with $g=T_{y_{0}} f \in \Lambda \operatorname{GBF}^{(p)}\left(G_{N_{0}}\right)$ whose Fourier series also has gaps (2.2).

Let $M \in \mathbb{N}$ be fixed such that $n_{M} \geq m_{N_{0}}$ and let $N \in \mathbb{N}$ be the integer such that $m_{N} \leq n_{M}<m_{N+1}$. Then clearly $N \geq N_{0}$. Put $t_{N}=m_{N} / m_{N_{0}}$ and for each $\alpha=0,1, \ldots, t_{N}-1, h \in G_{N}$, define

$$
f_{\alpha}(x)=f\left(x+z_{\alpha}^{(N)}+h\right)-f\left(x+z_{\alpha}^{(N)}\right), \quad x \in G .
$$

Then for all $n \geq 0$ we have

$$
\hat{f}_{\alpha}(n)=\hat{f}(n) \chi_{n}\left(z_{\alpha}^{(N)}+h\right)-\hat{f}(n) \chi_{n}\left(z_{\alpha}^{(N)}\right)=\hat{f}(n) \chi_{n}\left(z_{\alpha}^{(N)}\right)\left(\chi_{n}(h)-1\right) .
$$

Since $f \in \Lambda \mathrm{GBF}^{(p)}\left(G_{N_{0}}\right)$ for any $x \in G_{N_{0}}$ we have

$$
\begin{aligned}
|f(x)|^{p} & =|f(0)+f(x)-f(0)|^{p} \\
& \leq 2^{p}|f(0)|^{p}+2^{p}|f(x)-f(0)|^{p} \\
& =2^{p}|f(0)|^{p}+2^{p} \lambda_{1}\left(\frac{|f(x)-f(0)|^{p}}{\lambda_{1}}\right) \\
& \leq 2^{p}|f(0)|^{p}+2^{p} \lambda_{1}\left(\frac{\left(\operatorname{osc}\left(f ; z_{0}^{(0)}+G_{N_{0}}\right)\right)^{p}}{\lambda_{1}}\right) \\
& \leq 2^{p}|f(0)|^{p}+2^{p} \lambda_{1}\left(\Lambda G F_{p}\left(f ; G_{N_{0}}\right)\right)^{p} .
\end{aligned}
$$

Thus $f$ is bounded on $G_{N_{0}}=I$ and hence $f \in L^{2}(I)$. In view of (4.1) for $r=s=2$, $f \in L^{2}(G)$ and hence each $f_{\alpha} \in L^{2}(I)$. Since the Fourier series of $f_{\alpha}$ also has gaps (2.2), again using (4.1) for $f_{\alpha}$ and for $r=s=2$ (since $\left|\chi_{n}\left(z_{\alpha}^{(N)}\right)\right|=1$ ) we get

$$
B(h) \equiv \sum_{k=1}^{\infty}\left|\hat{f}\left(n_{k}\right)\right|^{2}\left|\chi_{n_{k}}(h)-1\right|^{2} \leq|I|^{-2}\left\|f_{\alpha}\right\|_{2, I}^{2}, \quad \text { for all } \alpha \text {. }
$$


Now, suppose $r>1$ and set $2=\frac{p+(2-p) s}{s}+\frac{p}{r}$; then using the Hölder's inequality we get

$$
\begin{aligned}
\left\|f_{\alpha}\right\|_{2, I}^{2} & =\int_{I}\left|f_{\alpha}(x)\right|^{2} d x \\
& =\int_{I}\left|f_{\alpha}(x)\right|^{\left(\frac{p+(2-p) s}{s}+\frac{p}{r}\right)} d x \\
& =\int_{I}\left(\left|f_{\alpha}(x)\right|^{(p+(2-p) s)}\right)^{1 / s}\left(\left|f_{\alpha}(x)\right|^{p}\right)^{1 / r} d x \\
& \leq\left\{\int_{I}\left|f_{\alpha}(x)\right|^{(p+(2-p) s)} d x\right\}^{1 / s}\left\{\int_{I}\left|f_{\alpha}(x)\right|^{p} d x\right\}^{1 / r} \\
& \leq\left(\Omega_{N}\right)^{1 / r}\left(\int_{I}\left|f_{\alpha}(x)\right|^{p} d x\right)^{1 / r},
\end{aligned}
$$

where $\Omega_{N}=\left(\omega^{(p+(2-p) s)}(f, N, I)\right)^{2 r-p}$ since $h \in G_{N}$. This together with (4.2) implies

$$
(B(h))^{r} \leq|I|^{-2 r} \Omega_{N} \int_{I}\left|f_{\alpha}(x)\right|^{p} d x,
$$

for all $\alpha=0,1, \ldots, t_{N}-1$. Since the left hand side of (4.3) is independent of $\alpha$, multiplying both sides of it by $\left(1 / \lambda_{\alpha+1}\right)$ and taking summation over $\alpha$, we get

$$
(B(h))^{r} \theta_{t_{N}} \leq|I|^{-2 r} \Omega_{N} \int_{I}\left(\sum_{\alpha=0}^{t_{N}-1} \frac{\left|f_{\alpha}(x)\right|^{p}}{\lambda_{\alpha+1}}\right) d x,
$$

where $\theta_{t}=\sum_{j=1}^{t}\left(1 / \lambda_{j}\right)=\sum_{j=0}^{t-1}\left(1 / \lambda_{j+1}\right)$, for all $t \in \mathbb{N}$; and hence

$$
B(h) \leq|I|^{-2}\left(\frac{\Omega_{N}}{\theta_{t_{N}}}\right)^{1 / r}\left\{\int_{I}\left(\sum_{\alpha=0}^{t_{N}-1} \frac{\left|f_{\alpha}(x)\right|^{p}}{\lambda_{\alpha+1}}\right) d x\right\}^{1 / r} .
$$

Integrating both sides of this inequality over $G_{N}$ with respect to $h$ we get

$$
\int_{G_{N}} B(h) d h \leq|I|^{-2}\left(\frac{\Omega_{N}}{\theta_{t_{N}}}\right)^{1 / r} \int_{G_{N}}\left\{\int_{I} \sum_{\alpha=0}^{t_{N}-1} \frac{\left|f_{\alpha}(x)\right|^{p}}{\lambda_{\alpha+1}} d x\right\}^{1 / r} d h .
$$

Now, for any $h \in G_{N}$ and any $x \in I=G_{N_{0}}$ the points $x+z_{\alpha}^{(N)}+h$ and $x+z_{\alpha}^{(N)}$ lie in the coset $x+z_{\alpha}^{(N)}+G_{N}$ of $G_{N}$ in $G_{N_{0}}$ (since $N \geq N_{0}$ ) and hence

$$
\left|f_{\alpha}(x)\right|=\left|f\left(x+z_{\alpha}^{(N)}+h\right)-f\left(x+z_{\alpha}^{(N)}\right)\right| \leq \operatorname{osc}\left(f, x+z_{\alpha}^{(N)}+G_{N}\right) .
$$

Since $f \in \Lambda G B F^{(p)}(I)$, for $h \in G_{N}$, in view of (4.5),

$$
\sum_{\alpha=0}^{t_{N}-1} \frac{\left|f_{\alpha}(x)\right|^{p}}{\lambda_{\alpha+1}} \leq \sum_{\alpha=0}^{t_{N}-1} \frac{\left(\operatorname{osc}\left(f, x+z_{\alpha}^{(N)}+G_{N}\right)\right)^{p}}{\lambda_{\alpha+1}} \leq\left(\Lambda G F_{p}(f ; I)\right)^{p}
$$


for all $x \in I$; because for any $x \in I$, the finite sequence of cosets $\left\{x+z_{\alpha}^{(N)}+G_{N}: \alpha=\right.$ $\left.0,1, \ldots, t_{N}-1\right\}$ is a rearrangement of the sequence $\left\{z_{\alpha}^{(N)}+G_{N}: \alpha=0,1, \ldots, t_{N}-1\right\}$ since this collection gives all the cosets of $G_{N}$ in $I$. Further, since $m_{N} \leq n_{M}<m_{N+1}$ and $n_{k} \geq n_{M}$ for $k \geq M$, from (4.2), we have

$$
\begin{aligned}
\int_{G_{N}} B(h) d h & \geq \sum_{k=M}^{\infty}\left|\hat{f}\left(n_{k}\right)\right|^{2} \int_{G_{N}}\left|\chi_{n_{k}}(h)-1\right|^{2} d h \\
& =\left(\frac{2}{m_{N}}\right) \sum_{k=M}^{\infty}\left|\hat{f}\left(n_{k}\right)\right|^{2}
\end{aligned}
$$

for all $\alpha$; in view of Lemma 4.1. Using (4.6) and (4.7) in (4.4) we get

$$
\begin{aligned}
R_{n_{M}} & \equiv \sum_{n=n_{M}}^{\infty}|\hat{f}(n)|^{2} \\
& =\sum_{k=M}^{\infty}\left|\hat{f}\left(n_{k}\right)\right|^{2} \\
& \leq|I|^{-2}\left(\frac{m_{N}}{2}\right)\left(\frac{\Omega_{N}}{\theta_{t_{N}}}\right)^{1 / r} \int_{G_{N}}\left\{\int_{I}\left(\Lambda G F_{p}(f ; I)\right)^{p} d x\right\}^{1 / r} d h \\
& =|I|^{-2}\left(\frac{m_{N}}{2}\right)\left(\frac{\Omega_{N}}{\theta_{t_{N}}}\right)^{1 / r}\left(\frac{\left(\Lambda G F_{p}(f ; I)\right)^{p}}{m_{N_{0}}}\right)^{1 / r}\left(\frac{1}{m_{N}}\right) \\
& =O\left[\left(\frac{\Omega_{N}}{\theta_{t_{N}}}\right)^{1 / r}\right]
\end{aligned}
$$

Now, applying Lemma 4.2 with $u_{k}=\left|\hat{f}\left(n_{k}\right)\right|^{2}$ and $F(u)=u^{\beta / 2}$ we get

$$
\sum_{k=1}^{\infty}\left|\hat{f}\left(n_{k}\right)\right|^{\beta}=\sum_{k=1}^{\infty} F\left(u_{k}\right) \leq 2 \sum_{k=1}^{\infty} F\left(\frac{1}{k} \sum_{j=k}^{\infty}\left|\hat{f}\left(n_{j}\right)\right|^{2}\right)=2 \sum_{k=1}^{\infty}\left(\frac{R_{n_{k}}}{k}\right)^{\beta / 2} .
$$

Therefore in view of (4.8) we obtain

$$
\begin{aligned}
\sum_{k=1}^{\infty}\left|\hat{f}\left(n_{k}\right)\right|^{\beta} & =O(1) \sum_{k=1}^{\infty}\left(\frac{R_{n_{k}}}{k}\right)^{\beta / 2} \\
& =O(1) \sum_{n=0}^{\infty} \sum_{\substack{k \\
m_{n} \leq n_{k}<m_{n+1}}}\left(\frac{R_{n_{k}}}{k}\right)^{\beta / 2} \\
& =O(1)\left\{1+\sum_{n=N_{0}}^{\infty} \sum_{\substack{k \\
m_{n} \leq n_{k}<m_{n+1}}}\left[\frac{\left(\Omega_{n}\right)^{1 / r}}{k\left(\theta_{t_{n}}\right)^{1 / r}}\right]^{\beta / 2}\right\}
\end{aligned}
$$




$$
=O(1)\left\{1+\sum_{n=N_{0}}^{\infty}\left[\frac{\left(\Omega_{n}\right)^{1 / r}}{\left(\theta_{t_{n}}\right)^{1 / r}}\right]^{\beta / 2} \sum_{\substack{k \\ m_{n} \leq n_{k}<m_{n+1}}} \frac{1}{k^{\beta / 2}}\right\}<\infty,
$$

by the assumption of theorem. Thus the theorem is proved for $r>1$.

For the case $r=1, s=\infty$, simply note that

$$
\left|f_{\alpha}(x)\right|^{2}=\left|f_{\alpha}(x)\right|^{2-p}\left|f_{\alpha}(x)\right|^{p} \leq\left(\omega_{N}(f, I)\right)^{2-p}\left|f_{\alpha}(x)\right|^{p}
$$

because

$$
\left|f_{\alpha}(x)\right|=\left|f\left(x+z_{\alpha}^{(N)}+h\right)-f\left(x+z_{\alpha}^{(N)}\right)\right| \leq \omega_{N}(f, I)
$$

for $x \in I$ and $h \in G_{N}$; and proceed as above.

Proof of Corollary 3.1. Taking $n_{k}=k$ for all $k, N_{0}=0, I=G$, and using the relation $\sum_{m_{n}}^{m_{n+1}-1} k^{-\beta / 2}=O\left(m_{n}^{1-\beta / 2}\right), n \in \mathbb{Z}_{+}$, we easily deduce Corollary 3.1 from Theorem 3.1 .

Proof of Theorem 3.2. As in the proof of Theorem 3.1, here also we may assume that $y_{0}=0$. Since $f \in \phi \Lambda \operatorname{GBF}(I)$ for any $x \in I=G_{N_{0}}$, we have

$$
|f(x)| \leq|f(0)|+C \phi^{-1}\left(\Lambda G F_{\phi}(f ; I)\right) .
$$

Thus $f$ is bounded on $I$ and hence $f \in L^{2}(I)$. For $r>1$, proceeding as in the proof of Theorem 3.1 we get (4.3). Since multiplying $f$ by a positive constant alters $\omega^{(p)}(f, n, I)$ by the same constant, and $\phi$ is $\Delta_{2}$, we may assume that $|f(x)| \leq \frac{1}{2}$ for all $x \in I$. Therefore we get $0 \leq\left|f_{\alpha}(x)\right|=\left|f\left(x+z_{\alpha}^{(N)}+h\right)-f\left(x+z_{\alpha}^{(N)}\right)\right| \leq 1$ and hence, as $p \geq 1$, it follows that $\left|f_{\alpha}(x)\right|^{p} \leq\left|f_{\alpha}(x)\right|$. Now, in view of (4.3) we get

$$
(B(h))^{r} \leq|I|^{-2 r} \Omega_{N} \int_{I}\left|f_{\alpha}(x)\right|^{p} d x \leq|I|^{-2 r} \Omega_{N} \int_{I}\left|f_{\alpha}(x)\right| d x, \quad \alpha=0,1, \ldots, t_{N}-1 .
$$

Since $\phi(2 x) \leq d \phi(x)$, for all $x \geq 0$, it follows (see, for example, [4, Proof of Theorem 2]) that

$$
\phi(a x) \leq d^{\log _{2} a+1} \phi(x), \quad \text { for all } x \geq 0 \text { and for all } a \geq 1 .
$$

Since $|I|^{-2 r} \Omega_{N} \geq 0$, if $|I|^{-2 r} \Omega_{N}<1$ then we get

$$
\begin{aligned}
\phi\left(m_{N_{0}}(B(h))^{r}\right) & \leq \phi\left(m_{N_{0}}|I|^{-2 r} \Omega_{N} \int_{I}\left|f_{\alpha}(x)\right| d x\right) \\
& \leq|I|^{-2 r} \Omega_{N} \phi\left(m_{N_{0}} \int_{I}\left|f_{\alpha}(x)\right| d x\right) .
\end{aligned}
$$

Further when $|I|^{-2} \Omega_{N} \geq 1$, in view of (4.10), we have

$$
\begin{aligned}
\phi\left(m_{N_{0}}(B(h))^{r}\right) & \leq \phi\left(m_{N_{0}}|I|^{-2 r} \Omega_{N} \int_{I}\left|f_{\alpha}(x)\right| d x\right) \\
& \leq d^{\log _{2}\left(|I|^{-2 r} \Omega_{N}\right)+1} \cdot \phi\left(m_{N_{0}} \int_{I}\left|f_{\alpha}(x)\right| d x\right)
\end{aligned}
$$




$$
\begin{aligned}
& =d \cdot\left(|I|^{-2 r} \Omega_{N}\right)^{\log _{2} d} \cdot \phi\left(m_{N_{0}} \int_{I}\left|f_{\alpha}(x)\right| d x\right) \\
& =d \cdot|I|^{-2 r \log _{2} d} \cdot\left(\Omega_{N}\right)^{\log _{2} d-1} \cdot \Omega_{N} \cdot \phi\left(m_{N_{0}} \int_{I}\left|f_{\alpha}(x)\right| d x\right) \\
& \leq d \cdot|I|^{-2 r \log _{2} d} \cdot \Omega_{N} \cdot \phi\left(m_{N_{0}} \int_{I}\left|f_{\alpha}(x)\right| d x\right),
\end{aligned}
$$

in view of the fact that $\left(\Omega_{N}\right)^{\log _{2} d-1} \leq 1$, as $|f(x)| \leq \frac{1}{2}$, for all $x \in I$, and $\log _{2} d-1 \geq 0$. Therefore in either case

$$
\phi\left(m_{N_{0}}(B(h))^{r}\right)=O(1) \Omega_{N} \phi\left(m_{N_{0}} \int_{I}\left|f_{\alpha}(x)\right| d x\right)=O(1) \Omega_{N} m_{N_{0}} \int_{I} \phi\left(\left|f_{\alpha}(x)\right|\right) d x,
$$

in view of the Jensen's inequality. Now multiplying both the sides of this inequality by $\left(1 / \lambda_{\alpha+1}\right)$ and taking summation over $\alpha=0,1, \ldots, t_{N}-1$ we get

$$
\phi\left(m_{N_{0}}(B(h))^{r}\right)=O(1)\left(\frac{\Omega_{N}}{\theta_{t_{N}}}\right) \int_{I}\left(\sum_{\alpha=0}^{t_{N}-1} \frac{\phi\left(\left|f_{\alpha}(x)\right|\right)}{\lambda_{\alpha+1}}\right) d x,
$$

where in the right-hand side we have denoted the constant ' $O(1) m_{N_{0}}$ ' by ' $O(1)$ ' itself. Since $f \in \phi \Lambda \operatorname{GBF}(I)$ and $\phi$ is increasing, for all $h \in G_{N}$ and $x \in I$ we have

$$
\sum_{\alpha=0}^{t_{N}-1} \frac{\phi\left(\left|f_{\alpha}(x)\right|\right)}{\lambda_{\alpha+1}} \leq \sum_{\alpha=0}^{t_{N}-1} \frac{\phi\left(\operatorname{osc}\left(f ; x+z_{\alpha}^{(N)}+G_{N}\right)\right)}{\lambda_{\alpha+1}} \leq \Lambda G F_{\phi}(f ; I) .
$$

Using (4.12) in (4.11) we get

$$
\phi\left(m_{N_{0}}(B(h))^{r}\right) \leq C\left(\frac{\Omega_{N}}{\theta_{t_{N}}}\right)
$$

where $C$ is a constant such that $C \geq 1$. Thus

and therefore

$$
m_{N_{0}}(B(h))^{r} \leq \phi^{-1}\left\{C\left(\frac{\Omega_{N}}{\theta_{t_{N}}}\right)\right\} \leq C \phi^{-1}\left(\frac{\Omega_{N}}{\theta_{t_{N}}}\right)
$$

$$
B(h)=O\left[\left\{\phi^{-1}\left(\frac{\Omega_{N}}{\theta_{t_{N}}}\right)\right\}^{1 / r}\right] .
$$

Integrating both sides of this inequality over $G_{N}$ with respect to $h$, in view of (4.7) we get

$$
R_{n_{M}} \equiv \sum_{k=M}^{\infty}\left|\hat{f}\left(n_{k}\right)\right|^{2} \leq\left(\frac{m_{N}}{2}\right) \int_{G_{N}} B(h) d h=O\left[\left\{\phi^{-1}\left(\frac{\Omega_{N}}{\theta_{t_{N}}}\right)\right\}^{1 / r}\right]
$$

Thus from (4.9) we get

$$
\sum_{k=1}^{\infty}\left|\hat{f}\left(n_{k}\right)\right|^{\beta}=O(1) \sum_{k=1}^{\infty}\left(\frac{R_{n_{k}}}{k}\right)^{\beta / 2}=O(1) \sum_{n=0}^{\infty} \sum_{\substack{k \\ m_{n} \leq n_{k}<m_{n+1}}}\left(\frac{R_{n_{k}}}{k}\right)^{\beta / 2}
$$




$$
\begin{aligned}
& =O(1)\left\{1+\sum_{n=N_{0}}^{\infty} \sum_{\substack{k \\
m_{n} \leq n_{k}<m_{n+1}}}\left[\frac{1}{k}\left\{\phi^{-1}\left(\frac{\Omega_{n}}{\theta_{t_{n}}}\right)\right\}^{1 / r}\right]^{\beta / 2}\right\} \\
& =O(1)\left\{1+\sum_{n=N_{0}}^{\infty}\left[\left\{\phi^{-1}\left(\frac{\Omega_{n}}{\theta_{t_{n}}}\right)\right\}^{1 / r}\right]_{\substack{k \\
m_{n} \leq n_{k}<m_{n+1}}}^{\beta / 2} \frac{1}{k^{\beta / 2}}\right\}<\infty,
\end{aligned}
$$

in view of the assumption of the theorem. This completes the proof of the theorem for $r>1$. For the case $r=1, s=\infty$, the proof is similar as that of Theorem 3.1.

Proof of Corollary 3.2. Similar as the proof of Corollary 3.1.

Acknowledgements. The author thanks Professor J. R. Patadia for his help and guidance in the preparation of this paper.

\section{REFERENCES}

[1] N. K. Bary, A Treatise on Trigonometric Series, Vols. I and II, New York: Pergamon, 1964.

[2] H. E. Chrestenson, A class of generalized Walsh functions, Pacific J. Math. 5 (1955), 17-31.

[3] N. J. Fine, On the Walsh functions, Trans. Amer. Math. Soc. 65 (1949), 372-414.

[4] B. L. Ghodadra, Applications of Hölder's and Jensen's inequalities in studying the $\beta$-absolute convergence Vilenkin-Fourier series, Math. Inequal. Appl. 17 (2) (2014), 749-760.

[5] B. L. Ghodadra, On the coefficients of Vilenkin-Fourier series with small gaps, Kyoto J. Math. 51 (4) (2011), 891-900.

[6] B. L. Ghodadra and J. R. Patadia, Wiener-Ingham type inequality for Vilenkin groups and its application to Harmonic Analysis, Period. Math. Hungar. 72 (1) (2016), 50-63.

[7] E. Hewitt and K. A. Ross, Abstract Harmonic Analysis, Vol. I, 2nd ed., Grundlehren Math. Wiss. 115, Springer, Berlin, 1979.

[8] C. W. Onneweer, Absolute convergence of Fourier series on certain groups, Duke Math. J. 39 (4) (1972), 599-609.

[9] C. W. Onneweer, Absolute convergence of Fourier series on certain groups, II, Duke Math. J. 41 (3) (1974), 679-688.

[10] C. W. Onneweer and D. Waterman, Fourier series of functions of Harmonic bounded fluctuation on groups, J. Analyse Math. 27 (1974), 79-93.

[11] C. W. Onneweer and D. Waterman, Uniform convergence of Fourier series on groups, I, Michigan Math. J. 18 (1971), 265-273.

[12] J. R. Patadia and R. G. Vyas, Fourier series with small gaps and functions of generalized variations, J. Math. Anal. Appl. 182 (1) (1994), 113-126.

[13] R. E. A. C. Payley, A remarkable series of orthogonal functions, Proc. London Math. Soc. 34 (1932), 241-279.

[14] T. S. Quek and L. Y. H. Yap, Absolute convergence of Vilenkin-Fourier series, J. Math. Anal. Appl. 74 (1980), 1-14.

[15] T. S. Quek and L. Y. H. Yap, Factorization of Lipschitz functions and absolute convergence of Vilenkin-Fourier series, Monatsch. für Math. 92 (1981), 221-229.

[16] M. Schramm and D. Waterman, Absolute convergence of Fourier series of functions of $\Lambda B V^{(p)}$ and $\phi \Lambda B V$, Acta. Math. Acad. Sci. Hungar. 40 (1982), 273-276. 
[17] S. B. Stečhkin, On the absolute convergence of orthogonal series - I, Trans. Amer. Math. Soc. 3 (1962), 271-280.

[18] S. B. Stečkin, On the theorem of Kolmogorov-Seliverstov, Izv. Akad. Nauk SSSR Ser Mat. 17 (1953), 499-512.

[19] O. Szász, Ueber die Fourierschen Reihen gewisser Funktionenklassen, Math. Ann. 100 (1928), $530-536$.

[20] Y. Uno, Absolute convergence of Vilenkin-Fourier series, Sci. Rep. Kanazawa Univ. 29 (2) (1984), 97-102.

[21] N. Ja. Vilenkin, On a class of complete orthonormal systems, Amer. Math. Soc. Transl. 28 (1963), 1-35.

[22] N. Ja. Vilenkin and A. I. Rubinstěin, A theorem of S. B. Stečhkin on absolute convergence of series with respect to systems of characters on zero-dimensional abelian groups, Izv. Vyš̌. Učebn. Zaved. Matematika 19 (9) (1975), 3-9 (in Russian); English Transl.: Soviet Math. 19 (1976), $1-6$.

[23] R. G. Vyas, On the absolute convergence of small gaps Fourier series of functions of $\Lambda B V^{(p)}$, JIPAM. J. Inequal. Pure Appl. Math. 6 (1) (2005), Art. 23.

[24] R. G. Vyas, On the absolute convergence of small gaps Fourier series of functions of $\varphi \Lambda B V$, JIPAM. J. Inequal. Pure Appl. Math. 6 (4) (2005), Art. 94.

[25] A. Zygmund, Trigonometric series, Vol. I and II combined, Cambridge University Press, 2002.

${ }^{1}$ Department of Mathematics,

FaCUlty of SCIENCE,

The Maharaja Sayajirao University of Baroda,

VADODARA - 390002 ,

GUJARAT, INDIA

E-mail address: bhikhu_ghodadra@yahoo.com 